\title{
Regularized OSIRIS 3D spectroscopy at the circumnuclear disk ionization front
}

\author{
Thibaut Paumard ${ }^{1}$, Mark R. Morris ${ }^{2}$, Tuan Do $^{3}$ and Andrea Ghez ${ }^{2}$ \\ ${ }^{1}$ LESIA-Observatoire de Paris, CNRS, UPMC Univ Paris 06, Univ. Paris-Diderot \\ 5 place Jules Janssen, F-92190 Meudon, France. email: thibaut.paumard@obspm.fr \\ ${ }^{2}$ Department of Physics and Astronomy, UCLA, Los Angeles, CA 90095-1547, USA \\ ${ }^{3}$ Dunlap Institute for Astronomy and Astrophysics, University of Toronto, \\ 50 St. George Street, Toronto M5S 3H4, ON, Canada
}

\begin{abstract}
The Galactic central black hole and the central cluster of young stars are surrounded by a clumpy gas disk (the circumnuclear disk, CND) that rotates about them at a distance of $\simeq 1 \mathrm{pc}$. The gas in this warm, turbulent, magnetized disk is ultimately likely to migrate into the central cavity and fuel future star formation and black hole accretion. We have observed two fields of approximately $20^{\prime \prime} \times 20^{\prime \prime}$ in the CND at NIR wavelengths with the OSIRIS integralfield spectrometer at Keck Observatory. These two fields are located at the interface between the neutral and the ionized regions. Our data cover two $\mathrm{H}_{2}$ lines as well as the $\mathrm{Br} \gamma$ line of the $\mathrm{HI}$ spectrum. The signal-to-noise ratio per spatial pixel of each line varies considerably across the field and becomes quite low in some regions. In order to avoid degrading the spatial resolution, we have developed a novel three-dimensional method to analyze the data in terms of regularized parameter maps. The method proves very efficient in retrieving all the information contained in the spectral line emission while not degrading the spatial resolution. We present this new method to analyze 3D spectroscopic data and describe our preliminary results on the structure of the ionized-neutral interface.
\end{abstract}

Keywords. methods: data analysis, techniques: spectroscopic, ISM: kinematics and dynamics.

\section{Introduction}

The circumnuclear disk is a well-defined ring of gas and dust orbiting the Galactic black hole and the central cluster of massive young stars. The importance of the CND is that it is a relatively massive reservoir of gas that will probably fuel future episodes of star formation and of strong accretion onto the central black hole, and in a previous phase of its existence, it may have been responsible for forming the present cluster of massive young stars (Morris \& Ghez 1996; Morris, Ghez \& Becklin 1999).

The CND is a warm (a few $100 \mathrm{~K}$ ) molecular medium characterized by strong turbulence, and clumpiness (Güsten et al. 1987; Genzel 1989; Marr et al. 1993; Jackson et al. 1993; Bradford et al. 2005). Indeed, the volume filling factor of dense material has been estimated to be as small as $1 \%$ (Vollmer \& Duschl 2001). The clumpiness seen in line emission from HCN and CS has led Christopher et al. (2005) and Montero-Castano et al. (2009) to conclude that the clumps must be self-gravitating, and thus particularly dense and massive in order to persist in the face of the strong tidal shear occurring this close to the central black hole. Their density estimates exceed $10^{8} \mathrm{~cm}^{-3}$ and the individual clump masses range up to several $\times 10^{5} \mathrm{M}_{\odot}$, for a total mass exceeding $10^{6} \mathrm{M}_{\odot}$. This is far larger than had previously been suggested for the entire CND (a few $\times 10^{4} \mathrm{M}_{\odot}$, based on measurements of the column densities of molecular lines and dust (Vollmer \& Duschl 2002, and references therein). This discrepancy is therefore rather problematical, and has not been satisfactorily explained. There are two important sources for this uncertainty: 
the unknown size spectrum of clumps in the CND, and the distribution of clump densities. Theoretically, the clump size distribution is expected to extend down to well below one arcsecond (Vollmer \& Duschl 2001).

Clump morphology is another key issue that can be elucidated by our observations. The theoretical treatments of the clump characteristics to date all assume spherical clumps, but that assumption is valid only if the clumps are self-gravitating, which requires high densities and thus large masses. If, on the other hand, the clumps have lower masses, they are subject to tidal shear, so they are pulled into linear configurations. Indeed, the $\mathrm{H}_{2}$ morphologies crudely sampled with the Hubble observations (Yusef-Zadeh et al. 2001) suggest that some fraction of the emission is organized into filamentary configurations. The confirmation of filamentary clumps would imply an upper limit to their densities that is well below some of the recent estimates based on virial equilibrium.

\section{Observations and data analysis}

To address these questions, we have used OSIRIS (Larkin et al. 2006) with LASER guide star adaptive optics on 2010 May, 13th and 19th, and 2011 July, 24th and 25th, to observe two mosaics in the CND. The two fields are at the inner edge of the CND near the location of the two apparent nodes in the approximately circular CND orbit (Jackson et al. 1993). We have used the $0.1^{\prime \prime}$ pixel scale and the Kn3 filter. Each mosaic is composed of $4.8^{\prime \prime} \times 6.4^{\prime \prime}$ sub-fields. The North-Eastern (NE) field, near the receding node, is an approximately square field $20^{\prime \prime}$ on each side with a small $\left(<1^{\prime \prime}\right)$ horizontal gap $\simeq 4^{\prime \prime}$ south of its central point. The South-Western (SW) mosaic covers a rectangular area of $18^{\prime \prime} \times 14^{\prime \prime}$ near the approaching node. Initial data reduction was performed using the OSIRIS pipeline.

The spectral band covers two $\mathrm{H}_{2}$ lines (2.12 and $2.22 \mu \mathrm{m}$ ) and the $\mathrm{Br} \gamma$ line of the Hi spectrum. We are interested in retrieving spatial maps of three physical quantities for each line: line flux, velocity and velocity dispersion. The classical approach to this problem is to perform spectral model fitting on each individual spectrum in the field. While this method is well understood and yields good results on high signal-to-noise ratio $(\mathrm{S} / \mathrm{N})$ regions, it fails to properly detect line emission from lower $\mathrm{S} / \mathrm{N}$ regions. The reason is that, by fitting the spectra individually, one gives up important information contained in the data: since we are observing extended features and since the instrumental spatial resolution is finite, the results found on neighboring pixels should show correlations. In this context, it is customary to smooth the data in the spatial directions at the expense of spatial resolution, which is crucial in our case as explained in the introduction.

For this new study of the CND, we have designed an original approach which manages to preserve spatial resolution while fully using $2 \mathrm{D}$ information, preventing the fitting engine from fitting noise spikes and ensuring that the resulting parameter maps are smooth at tunable small scales. This is done by fitting the entire cube at once and using a compound estimator which is the sum of a classical $\chi^{2}$ term and a regularization term which ensures that the parameter maps are "smooth", or "regular" in the accepted terminology. The idea of regularization comes from the field of image deconvolution, which is very similar to our 3D fitting problem in that both are based on many-parameter fitting for which parameters are organized as images (or maps) that are known a priori to be smooth. We borrowed the L2-L1 regularization function from Yoda† by Damien Gratadour, a Yorick $\ddagger$ port of MISTRAL (Mugnier, Fusco \& Conan 2004).

$\dagger$ https://github.com/dgratadour/Yoda

$\ddagger$ http://yorick.github.com/ 

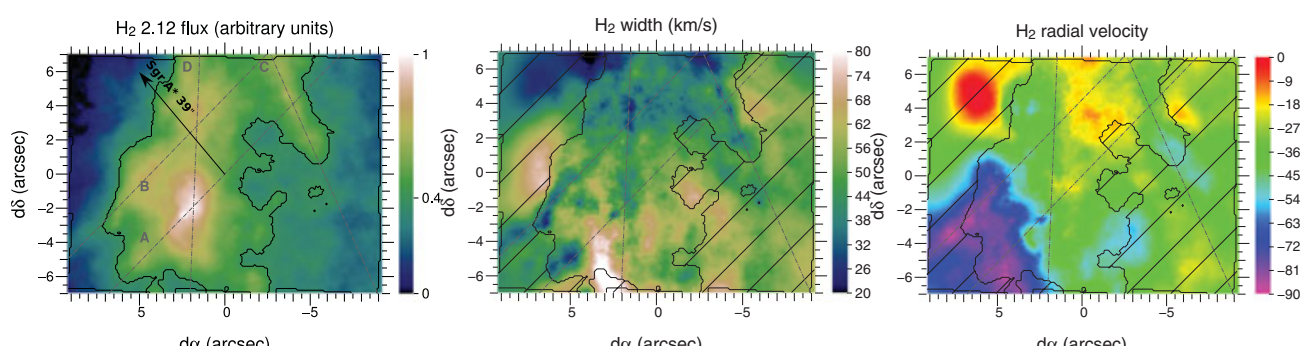

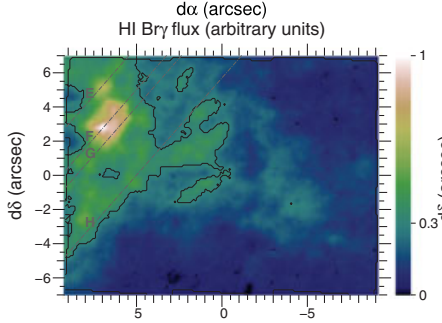

$\mathrm{d} \alpha(\operatorname{arcsec})$

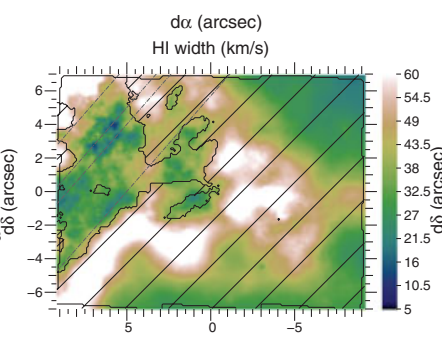

$\mathrm{d} \alpha(\operatorname{arcsec})$

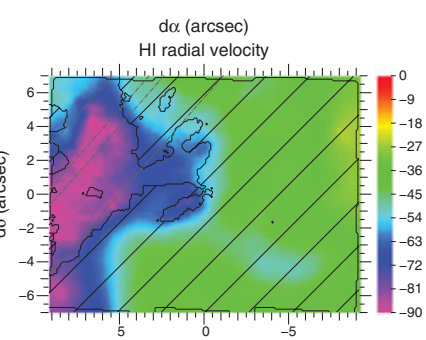

$\mathrm{d} \alpha(\operatorname{arcsec})$

Figure 1. SW mosaic, fitted maps. Top: $\mathrm{H}_{2}$. Bottom: $\mathrm{Hi} \mathrm{Br} \gamma$. Left to right: line flux $(2.12$ $\mu \mathrm{m}$ in the case of $\mathrm{H}_{2}$ ), width and Doppler shift. The last parameter (flux ratio between the two $\mathrm{H}_{2}$ lines) is not shown. Arrow: direction and distance to Sgr A*. Black lines: boundaries of the mosaic field-of-view. Black curve: flux limits $(S / N \simeq 3$ to 10 depending on the field and species) below which the width and velocity errors grow above useful limits $\left(10\right.$ to $\left.30 \mathrm{~km} \mathrm{~s}^{-1}\right)$. Those low $\mathrm{S} / \mathrm{N}$ regions are hashed out on these maps. Lettered gray lines: straight features, see text. The narrowest of these reconstructed features are only $\simeq 2.5$ pixel wide (FWHM). [A COLOR VERSION IS AVAILABLE ONLINE.]

\section{Results}

Our method was very successful in allowing detection of spectral lines even at very low signal-to noise and in creating parameter maps with high spatial resolution and little noise. Analysis and interpretation of those maps is ongoing and will be published soon (Paumard et al. 2014, in prep.). We do detect $\mathrm{H}_{2}$ almost throughout both fields, with local variations at all scales. The maps suggest a superposition of bulk motion with local variations. $\mathrm{Br} \gamma$ is bright only on the side of each field closest to $\mathrm{Sgr} \mathrm{A}^{*}$ and the central massive star cluster (Figures 3 and 1), although it is detected throughout the SW field.

Both fields exhibit compact features (small spots in the NE field, filamentary features in the SW field) with rather high line flux and relatively narrow line width. The line flux and width maps are however not strictly anti-correlated and we have checked by resorting to classical 1D spectral fitting that these variations are real and not some artifact of our 3D fitting procedure. We interpret these features as compact clumps with large optical thickness and low intrinsic velocity dispersion.

Several of the SW filaments are parallel to each other and mostly orthogonal to the direction towards Sgr A*. Those filaments have a distinctly highly negative radial velocity compared to the rest of the $\mathrm{H}_{2}$ emission in this field and this system of filaments extends closer to Sgr A* in HI, suggesting that the material emitting the two lines is physically related and that we are actually seeing the ionization front. In the NE field, the HI and $\mathrm{H}_{2}$ flux maps appear to be almost complementary, which is consistent with an ionization front seen edge-on. $\mathrm{S} / \mathrm{N}$ allows comparing $\mathrm{HI}$ and $\mathrm{H}_{2}$ velocities only in two small regions. While they are similar in the South-East corner of the field, they are significantly different (by $\simeq 50 \mathrm{~km} \mathrm{~s}^{-1}$ ) in the North-West part, making it unlikely that the $\mathrm{H}_{2}$ and $\mathrm{HI}$ lines 

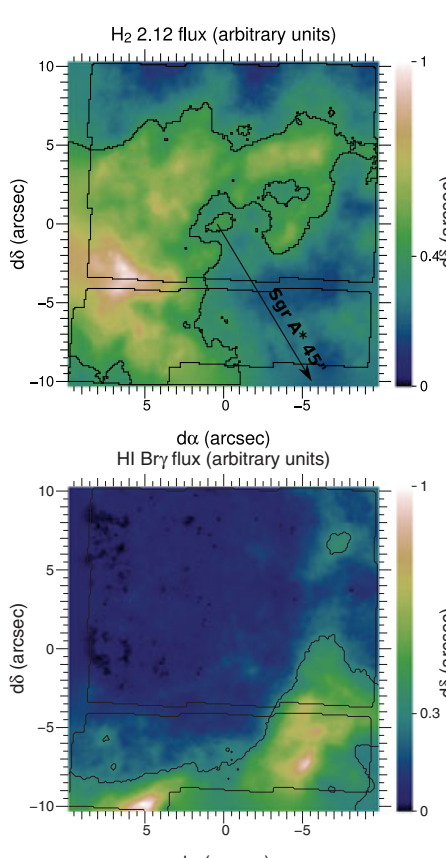

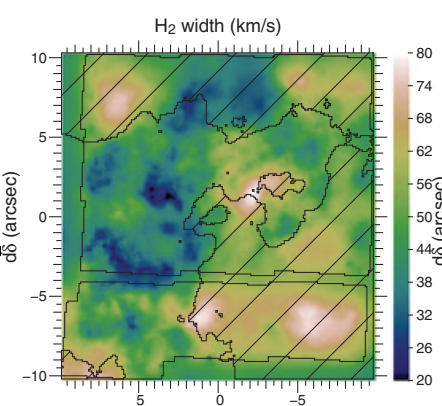

$\mathrm{d} \alpha(\operatorname{arcsec})$

$\mathrm{HI}$ width $(\mathrm{km} / \mathrm{s})$

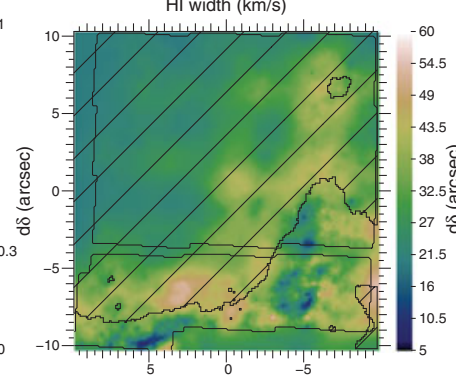

$\mathrm{d} \alpha(\operatorname{arcsec})$

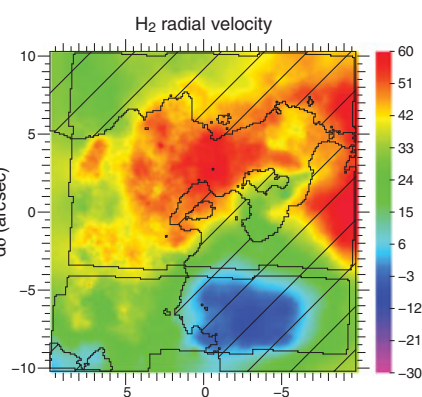

$\mathrm{d} \alpha(\operatorname{arcsec})$

$\mathrm{HI}$ radial velocity

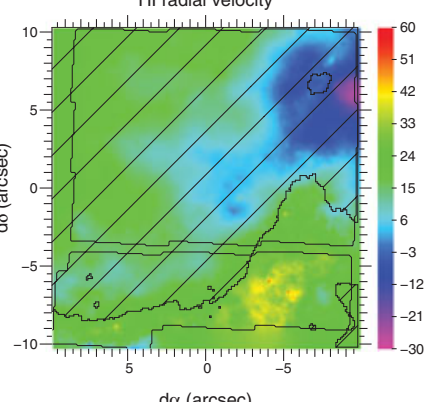

Figure 2. NE mosaic, same as Figure 1. [A COLOR version is AVAilable online.]

originate in the same clump there. For this part of the field, it appears more likely that we are seeing the alignment of two clumps, one of which is fully ionized.

\section{References}

Bradford, C. M., Stacey, G. J., Nikola, T., Bolatto, A. D., Jackson, J. M., Savage, M. L., \& Davidson, J. A. 2005, ApJ 623, 866

Burton, M. G., Hollenbach, D. J., \& Tielens, A. G. G.. M. 1990, ApJ 365, 620

Christopher, M. H., Scoville, N. Z., Stolovy, S. R., \& Yun, M. S. 2005, ApJ 622, 346

Gatley, I., Jones, T. J., Hyland, A. R., Wade, R., Geballe, T. R., \& Krisciunas, K. 1986, MNRAS 222,299

Genzel, R. 1989, in: M. R. Morris (ed.), The Center of the Galaxy, Proc. IAU Symposium No. 136 (Dordrecht: Kluwer), p. 393

Güsten, R., Genzel, R., Wright, M. C. H., et al. 1987, ApJ 318, 124

Jackson, J. M., Geis, N., Genzel, R., Harris, A. I., Madden, S., Poglitsch, A., Stacey, G. J., \& Townes, C. H. 1993, ApJ 402, 173

Larkin, J., Barczys, M., Krabbe, A., Adkins, S., Aliado, T., Amico, P., Brims, G., Campbell, R., Canfield, J., Gasaway, T., Honey, A., Iserlohe, C., Johnson, C., Kress, E., LaFreniere, D., Lyke, J., Magnone, K., Magnone, N., McElwain, M., Moon, J., Quirrenbach, A., Skulason, G., Song, I., Spencer, M., Weiss, J., \& Wright, S. 2006, in: I. S. McLean \& M. Iye (eds.), Ground-based and Airborne Instrumentation for Astronomy, Proc. SPIE, Volume 6269 (SPIE Press), p. 62691A

Marr, J. M., Wright, M. C. H., \& Backer, D. C. 1993, ApJ 411, 667

Montero-Castano, M., Herrnstein, R. M., \& Ho, P. T. P.. 2009, ApJ 695, 1477

Morris, M. R. \& Serabyn, A. 1996, ARAA 34, 645

Morris, M. R., Ghez, A., \& Becklin E. E. 1999, Adv. Sp. Res. 23, 959

Mugnier, L. M., Fusco, T., \& Conan, J. M. 2004, J Opt Soc Am A Opt Image Sci Vis 21, 1841

Paumard, T., Maillard, J. P., \& Morris, M. R. 2004, A\&\&A 426, 81 
Sternberg, A. \& Dalgarno, A. 1989, ApJ 338, 197

Vollmer, B. \& Duschl, W. J. 2001, A\& A 367, 72

Vollmer, B. \& Duschl, W. J. 2002, A\&GA 388, 128

Yusef-Zadeh, F., Stolovy, S. R., Burton, M., Wardle, M., \& Ashley, M. C. B. 2001, ApJ 560, 749 